\title{
Ängste in der Schwangerschaft
}

\author{
Dr. med. Dorothea Schuster, Dresden
}

\section{Weshalb und welche Art von Ängsten haben Schwangere, besonders nach Fehl- oder Totgeburt? Wie können Hebammen Schwangere mit Ängsten gut betreuen?}

Angst ist ein Grundgefühl des Menschen. Erwartete Bedrohungen, etwa körperliche Angriffe, aber auch Verletzungen der psychischen Integrität, des Selbstwertgefühls sowie Veränderungen der psychosozialen Stabilität lösen Ängste aus.

Ängste haben auch eine Schutzfunktion, die nicht nur für das Überleben unverzichtbar ist, sondern ebenso eine Anpassung an besondere Situationen ermöglicht. Sowohl äußere Bedrohungen, die von den Sinnesorganen wahrgenommen werden, als auch Informationen aus dem Körperinneren über dortige Veränderungen werden dabei als „Gefahrenmeldung“ an wichtige Hirnzentren (Thalamus, Amygdala) weitergeleitet und setzen über die Aktivierung der Stressachse (Hypothalamus-Hypophyse-Nebennierenrinde) eine Reihe physiologischer Prozesse in Gang (z.B. Ausschüttung des Stresshormons Cortisol), mit denen Kampf oder Flucht als normale Reaktionen auf die Bedrohung möglich werden [1].

Was uns in welchem Maße Angst macht, ist neben grundlegenden Persönlichkeitseigenschaften immer auch eine Sache der Erfahrung, des Erlebens und der Bedeutung; unsere Angstgeschichte wird durch unsere persönliche Biografie geschrieben. Neue Studien, besonders aus der Zwillingsforschung, zeigen, dass aber auch genetische Faktoren am Ausmaß von Angsterleben beteiligt sind [1].

\section{Ängste in der Schwanger- schaft}

Die Schwangerschaft stellt nach psychosomatischem Verständnis eine Schwellensituation dar, in der das bio-psychosoziale Gleichgewicht besonderen Anforderungen und Belastungen ausgesetzt ist. Die schwangere Frau erlebt nicht nur erst- und einmalig gravierende Veränderungen des Körperbildes, bedingt durch hormonelle Umstellungen und durch das heranwachsende Kind, sondern auch eine emotionale Labilisierung durch die Veränderung ihrer bisherigen Identität und die Erfahrung, dass sich Beziehungen und das soziale Umfeld weitreichend verändern $[2,3]$.

Dabei auftretende ambivalente Gefühle gehören zu jeder Schwangerschaft, denn diese bedeutet nicht nur eine wesentliche Erweiterung von Lebenserfahrung, von persönlicher Kompetenz und Selbstwertgefühl, sondern auch einen Abschied von der bisherigen Identität mit Verlusten von Unabhängigkeit und Autonomie.

Die Erfahrung, dass sich eine Schwangerschaft - beginnend mit der Empfängnis als einem überwiegend passivem Geschehen - unabhängig von den eigenen Bemühungen einstellt und vollzieht, löst bei vielen Frauen und Paaren Erstaunen und Freude aus. Diese Erfahrung kann aber auch verunsichern und ängstigen, weil sich die wesentlichen Entwicklungen der Schwangerschaft der eigenen Kontrolle und Machbarkeit entziehen.
Auch das sich entwickelnde Kind, das mit fortschreitender Schwangerschaft als eigenständiges Wesen wahrgenommen wird, muss nicht nur körperlich, sondern auch in psychodynamischer Hinsicht integriert werden.

Die meisten Frauen und Paare bewältigen diese Veränderungen ohne Probleme und ohne zusätzliche professionelle Hilfe.

Schwangerschaft und Geburt unterliegen heute, bedingt durch vielfältige Entwicklungen und Möglichkeiten der modernen Geburtsmedizin, einer hohen Sicherheit für Mutter und Kind. Die Erfahrung, dass es sich dennoch um existenzielle Lebensereignisse handelt, die sich eigenen Bedürfnissen nach Kontrolle und Selbstbestimmung nicht vollständig unterordnen lassen, tritt angesichts dieser Möglichkeiten nicht selten in den Hintergrund.

Ängste von Schwangeren vor

- Schwangerschaftskomplikationen,

- Fehl- oder Frühgeburt,

- Fehlbildungen und Totgeburt,

- der Geburt (Erleben, Bewältigung, Komplikationen),

- der Verantwortung als Schwangere und als Mutter,

- einem Versagen als Schwangere und als Mutter,

- negativen körperlichen Veränderungen,

- Veränderungen der Paarbeziehung 
Ahnungen davon bleiben bei aufmerksamer Wahrnehmung jedoch spürbar. Ängste und Befürchtungen zur Gesundheit des erwarteten Kindes, aber auch zum eigenen guten und gesunden Überleben und Bewältigen von Schwangerschaft und Geburt werden in jeder Schwangerschaft mehr oder weniger stark erlebt.

Mitunter äußern sich existenzielle Ängste in Träumen als Ausdruck intensiver Beschäftigung des Unbewussten mit den erwarteten Veränderungen und werden von den Frauen erst geäußert, wenn wir sie fragen. Ängste sollten weder pathologisiert werden noch sollte versucht werden, sie durch das Angebot zusätzlicher Untersuchungen vorschnell zu entkräften.

Nicht selten verschieben sich Ängste aber auch hin zu erheblich geringer erscheinenden Anlässen, z.B. vermutete Ernährungsfehler oder Verhaltensunsicherheiten und werden mitunter als Fragen formuliert, die auf den ersten Blick banal erscheinen.

Auch die inzwischen fast selbstverständliche Annahme vieler Schwangerer, dass eine Befreiung von der beruflichen Belastung sinnvoll sei, kann neben unzureichenden gesellschaftlichen Voraussetzungen für die Vereinbarkeit von Berufstätigkeit und Schwangerschaft auch als Hinweis auf eine kollektive Verunsicherung und als Ausdruck von Ängsten verstanden werden: eine weitgehend normale Belastungsfähigkeit in der Schwangerschaft scheint nicht mehr vorstellbar zu sein.

Deutlich mehr Ängste erleben verständlicherweise Schwangere, die Verlusterfahrungen aus vorherigen Schwangerschaften mitbringen [4].

Bereits nach einer normalen, unkomplizierten Schwangerschaft, die mit der Geburt eines gesunden Kindes endete, äußern Frauen mitunter, dass sie die neue Schwangerschaft nicht mehr so unbeschwert wie beim ersten Kind beginnen. Das kann darauf hindeuten, dass die
Erfahrung von Schwangerschaft und Geburt als existenziell erlebt wird (wie oben beschrieben). Die konkreten Erfahrungen mit Kindern und der veränderten Familienstruktur lassen erkennen, dass Lebenserweiterung immer auch Erweiterung von Verletzlichkeit und Risiken bedeutet.

\section{Nach Verlusterfahrungen}

Bestehen darüber hinaus Erfahrungen mit Fehl- und Totgeburten, ist die psychische Belastung in der Folgeschwangerschaft deutlich erhöht; als vorrangige Reaktionen auf eine Fehlgeburt werden häufigere Ängste und verstärkte Angstsymptome beschrieben $[5,6]$.

Allerdings sind auch Frauen mit solchen Verlusterfahrungen im überwiegenden Maß in der Lage, die Folgen ohne zusätzliche professionelle Hilfe zu bewältigen; eine stabile Paarbeziehung ist dabei eine wichtige Ressource [7].

Ein Teil von ihnen, ca. 20\% [4], entwickelt jedoch eine lang anhaltende pathologische Trauerreaktion oder depressive Symptome sowie anhaltende schwangerschaftsbezogene Ängste, die auch im Verlauf der Schwangerschaft nicht erheblich abnehmen. Das Risiko dafür steigt nachvollziehbar nach mehreren Fehlgeburten an [5].

\section{Umgang mit Ängsten in der Schwangerschaft}

Das Konzept des „tender loving care“ wurde für die Begleitung von Frauen nach Fehlgeburten entwickelt. Sinngemäß übersetzt bedeutet es ,in liebevoller Fürsorge begleiten“ und umfasst häufigere (z. B. wöchentliche) Konsultationen mit jeweils sonografischen und bedarfsweise weiteren Untersuchungen sowie unterstützende Gespräche. Außerdem werden Entspannungsmaßnahmen vermittelt.
Studien haben gezeigt, dass Frauen nach mehreren Fehlgeburten, die in der Folgeschwangerschaft nach diesem Konzept betreut wurden, häufiger erfolgreich ihre Kinder ausgetragen haben als Frauen, die in üblicher Weise betreut wurden $[5,8]$.

Wie im Fall von Frau T. gezeigt, besteht die Aufgabe der Betreuungspersonen (Hebamme, Arzt/Ärztin) neben der fachlichen Betreuung vorrangig darin, die Ängste der Schwangeren als normal zu akzeptieren, auszuhalten, mitzutragen und fachkundig, kompetent und empathisch zu begleiten [6]. Gelingt dies, kann die Beziehung zwischen der Schwangeren und ihrer Betreuungsperson als Ressource für die Stabilisierung der Schwangerschaft genutzt werden [9].

Vergleichbar ist diese Aufgabe mit einer Art „Container-Funktion“ (für die Ängste und für alle Emotionen der Schwangeren), die am besten gelingt, wenn die Betreuenden selbst einen Zustand „reflektierter Angstfreiheit“ erreichen und mit Ruhe die Schwangere mit ihren Emotionen auffangen und „aushalten“ können.

Neben professioneller Sicherheit und Berufserfahrung setzt dies das Wissen um und die Auseinandersetzung mit eigenen Ängste voraus sowie die Fähigkeit, die eigenen Ängste von denen der Schwangeren zu unterscheiden und abzugrenzen.

\section{Mögliche Ängste der Betreuenden}

[10]:

- vor dem Versagen, die Erwartungen an eine optimale Betreuung nicht zu erfüllen,

- vor der Enttäuschung der Schwangeren bei ungünstigem Verlauf/ Ausgang der Schwangerschaft mit drohendem Beziehungsabbruch,

- vor forensischen/juristischen Konsequenzen,

- vor dem Verlust des Selbstwertgefühls und der professionellen Identität 


\section{Fallbeispiel}

Frau T., eine 36-jährige Frau, berichtete bei ihrem 1. Besuch in der Praxis sichtlich bedrückt von der kürzlichen Fehlgeburt ihrer 1. Schwangerschaft in der 9. SSW.

Danach hatte sie den Arzt gewechselt, da er bei ihr eine „Angststörung“ vermutete und sie sich nicht verstanden fühlte. Die Schwangerschaft war nach einer knapp 1-jährigen Kinderwunschbehandlung spontan eingetreten. Seit der Fehlgeburt fühlte sie sich oft gereizt und niedergeschlagen. Sorgenvolle Gedanken und Grübeln erlebte sie in allen Lebensbereichen. In ihren Träumen tauchte oft das Thema Schwangerschaft auf. Auch körperlich fühlte sie sich schwanger, ohne dass eine Schwangerschaft bestätigt wurde.

Ihr Ehemann, der sie bei allen Arztbesuchen begleitete, nahm sich spürbar zurück und wurde von Frau T. als sehr verständnisund rücksichtsvoll beschrieben. Gleichzeitig spürte sie starke Schuldgefühle ihm gegenüber, da sie sich mit ihren Wünschen nach seiner Zuwendung wie „unersättlich“ fühlte und befürchtete, ihn damit zu überfordern. Ein angebotenes gemeinsames Gespräch, das üblicherweise zu Beginn jeder Kinderwunschbehandlung angeboten wird, wollte sie deshalb nur allein wahrnehmen.

Im Gespräch berichtete sie, dass sie den erwarteten Veränderungen durch ein Kind mit großen Ängsten gegenüberstehe, da ihr Sicherheit schon immer wichtig gewesen sei und alle neuen Situationen sie zunächst verunsichern und angstbesetzt seien. Als Hintergrund vermutete sie einen hohen Leistungsanspruch, mit dem sie aufgewachsen sei.

Die Zusicherung, dass wohl jede Frau in ihrer Situation an eine neue Schwangerschaft mit mehr Ängsten herangehen würde, entlastete sie offensichtlich etwas. Auch das Bild von einem Sicherheitsnetz, dass sich mit dem Prinzip des „tender loving care“ für Frauen nach Fehlgeburten während der nächsten Schwangerschaft als sinnvoll erwiesen hat, gefiel ihr.

Sechs Monate später wurde eine intakte Schwangerschaft in der 6. SSW festgestellt.

Ihre Freude darüber war deutlich eingeschränkt durch die starke Fokussierung auf die vorangegangene Fehlgeburt und durch das sorgenvolle Wahrnehmen aller normalen Körpersymptome einer Frühschwangerschaft. Die Ängste steigerten sich zur Verzweiflung, als in der 9. SSW erneut ein missed abortion festgestellt wurde.

Besonders der gleiche Zeitpunkt beider Fehlgeburten löste bei ihr das Gefühl einer „gesetzmäßigen Wiederholung“ aus, dem sie sich ohnmächtig ausgeliefert fühlte.

Die Botschaft ihrer behandelnden Ärztin, dass sie diese Sicht zwar verstehen könne, selbst aber nicht teile, sondern am „Prinzip Hoffnung“ festhalte, konnte sie zu dieser Zeit nicht nachvollziehen. Da sie von Erinnerungen an die erste Fehlgeburt bedrückend überflutet wurde und sich deutliche Zeichen einer depressiven Entwicklung zeigten, wurde eine psychotherapeutische Begleitung empfohlen und von ihr auch initiiert.

Parallel dazu erfolgten eine Thrombophiliediagnostik sowie eine genetische Beratung und Untersuchung des Paares, jeweils mit unauffälligen Ergebnissen. Diese wurden mit dem Paar besprochen und jeweils mit stützenden Interventionen im Rahmen der psychosomatischen Grundversorgung verbunden, ausgerichtet auf das „Prinzip Hoffnung“.

Überraschend schnell war Frau T. ein 1/4 Jahr später erneut schwanger. Selbstzweifel am guten Ausgang dieser Schwangerschaft und die Zuschreibung eigener Schuld am bisherigen Misslingen („es muss an mir liegen“) standen der zaghaften Hoffnung gegenüber.

In den zunächst wöchentlich vereinbarten Terminen wurde die Intaktheit der Schwangerschaft jeweils zuerst sonografisch bestätigt, bevor danach Raum für Fragen, Ängste und Sorgen war. Frau T. berichtete oft von bedrohlich erlebten „Bildern in Schwarz“. Diese Berichte wurden angehört, mit Gelassenheit als negative Gefühle akzeptiert, aber auch ergänzt durch andere Wahrnehmungen vom sich normal entwickelnden Embryo und von der gut fortschreitenden Schwangerschaft. Die Würdigung ihrer „Leistung“, alle diese anstrengenden Gefühle auszuhalten, gemeinsam mit ihrem Mann, der sie stets begleitete, sowie das Suchen nach praktischen Möglichkeiten, damit umzugehen (z.B. Tagebuchschreiben, Malen, angepasste Arbeitsstrukturen) und zu entspannen und die Selbstfürsorge zu stärken, ergänzten die Konsultationen.

Da Frau T. die angebotenen häufigen Termine sehr ambivalent erlebte, einerseits sehnlich erwartete, sich andererseits durch eine extrem starke Anspannung kurz davor noch mehr belastet fühlte, wurde eine flexible Terminwahrnehmung vereinbart, die ihr das Gefühl eigener Kompetenz und Kontrolle und den Wunsch, sich als „normale Schwangere“ zu fühlen, besser ermöglichte. Besonders um den Zeitpunkt der vorangegangenen Fehlgeburten war ihr eine engmaschige Begleitung dann wieder wichtig. Frau T. war außerdem sehr ambivalent gegenüber pränataldiagnostischen Angeboten eingestellt, was sich in ihrer Unsicherheit über das „richtige Maß“ an Ultraschall-Untersuchungen und über die Inanspruchnahme zusätzlicher Diagnostik (FTS, Fehlbildungsdiagnostik) zeigte und auch zu kurzzeitigen Missverständnissen bei Terminabsprachen und Überweisungen führte. Ihrem Bestreben, soviel wie möglich über ihr Kind zu erfahren und höchstmögliche Sicherheit zu gewinnen, stand ihr Wunsch gegenüber, ihr Kind nicht zu gefährden und durch Diagnostik in Frage zu stellen.

Die Bestätigung, dass viele Frauen und Paare die Entscheidung über die PND als schwierig und zwiespältig erleben sowie die Ermutigung, auf ihr Bauchgefühl zu hören, wirkte entlastend. Die parallele Hebammenbetreuung mit abwechselnden Terminen bei Hebamme und Ärztin im Rahmen der normalen Mutterschaftsvorsorge erlebte Frau T. als sehr hilfreich.

In der 21. SSW spürte Frau T. erstmals Kindsbewegungen. Dies war ein weiterer wichtiger Schritt zur Entspannung. Ihre Möglichkeit, zum Kind spürbaren Kontakt aufnehmen zu können und seine wechselnde Lebendigkeit wahrzunehmen, erlebte Frau T. als deutlichen Zuwachs an Sicherheit. Normale Terminabstände waren jetzt ausreichend und freudige Hoffnung stellte sich stärker ein, allerdings immer wieder begleitet von Selbstzweifeln und Ängsten, die aufgefangen und besprochen werden mussten. Gegenwärtig ist Frau T. in der 27. SSW. Die Schwangerschaft verläuft normal und deutlich entspannt. 
Besonders wenn die Betreuungsperson in der eigenen Biografie negative Schwangerschaftserfahrungen mit Fehl- und/ oder Totgeburten erlebt hat, kann es ohne Reflexion zur verstärkten Identifizierung mit der Schwangeren kommen und sich als Rollenkonflikt hinderlich auf die Betreuung auswirken.

Eine ähnliche „Containing-Funktion“ gilt für den Umgang mit den fast immer vorhandenen Schuldgefühlen der Schwangeren. Das Wissen der Schwangeren um diese Ängste und ihre erfolglose Anstrengung, diese um jeden Preis zu vermeiden, löst zusätzliche Schuld- und Schamgefühle aus, die das Erleben von „reproduktiver Insuffizienz“ im Vergleich mit anderen Frauen verstärkt. Überforderung und Anspannung sind dann ungünstige Folgen der Kompensationsversuche.

Die Akzeptanz und die ausgesprochene Wertschätzung ihrer Bemühungen und die Würdigung ihrer „Leistung“ (all diese intensiven Gefühle auszuhalten) durch die Betreuenden ermöglichen es der Schwangeren, ihr eigenes Fühlen und Erleben als normal einzuordnen und können damit deutlich entspannend wirken.

In diesem Sinne und zur Vermeidung unnötiger Pathologisierung sollten zusätzliche Interventionen sowohl verbaler als auch anderer Art (z. B. zusätzliche Untersuchungen, Akupunktur) sorgfältig abgewogen und nur sparsam eingesetzt werden. Meist fühlen sich Schwangere in ihrer Situation, die vom erwarteten Idealbild der stets glücklichen Schwangeren abweicht, in ihrer Normalität ohnehin verunsichert und gegenüber anderen Frauen weniger kompetent.

Sachliche Informationen über physiologische Schwangerschaftsverläufe, deren normale Varianten und die grundsätzlich positive Prognose auch nach Fehlgeburten tragen zur Entspannung bei; ebenso die Haltung der Betreuungsperson, Störungen im Verlauf der Schwangerschaft nicht als Scheitern zu sehen, sondern als individuelle Schwierigkeiten auf einem Weg, der noch zu gehen ist.

Auch wenn das Prinzip des „tender loving care" vorrangig für Schwangere mit habituellen Aborten entwickelt wurde, spricht nichts dagegen, das Grundprinzip der liebevollen Fürsorge auch für Schwangere zu praktizieren, die aufgrund anderer Belastungen erhebliche Ängste haben und die „Container-Funktion“ im Umgang mit diesen Ängsten zu beherzigen.

Besonders für Frauen mit mehrfachen Fehlgeburten und einem in Studien nachgewiesenen deutlich erhöhten Angstniveau während der gesamten Folgeschwangerschaft [5] hat sich darüber hinaus ein intensiviertes Betreuungskonzept mit zusätzlicher supportiver Psychotherapie als hilfreich erwiesen [9].

Wie bei Frau T. gezeigt, ist es offensichtlich günstig, wenn dieses Konzept innerhalb eines interdisziplinären Netzwerkes angeboten und umgesetzt werden kann [2]. Dabei arbeiten verschiedene Professionen ( Hebammen, Ärztinnen verschiedener Fachrichtungen, Psychologinnen, Stillberaterin, Physiotherapeutin, Sozialpädagogin) bei der Betreuung von Schwangeren und Wöchnerinnen mit psychischen Problemen in strukturierter Form zusammen und können, entgegen vielen anderen Versorgungsformen, bei Bedarf kurzfristige Hilfe ohne Wartezeit (auf Psychotherapie) anbieten und diese Hilfe untereinander abstimmen.

Diese Kooperation bewirkt nicht nur eine effektive zeitnahe Unterstützung der belasteten Schwangeren mit langfristigen positiven Auswirkungen (z.B. für die Bindung zwischen Mutter und Kind), sondern dient auch der eigenen Entlastung und der Psychohygiene, um die Begleitung mit Gelassenheit und Zuversicht zu leisten.

Auch wenn kein solches Netzwerk zur Verfügung steht, ist ein regelmäßiger Austausch der Betreuenden in Koopera- tionen (Hebamme/Ärztin) sinnvoll und hilfreich.

\section{Literatur}

1 Bandelow B (2013): Das Angstbuch. Woher Ängste kommen und wie man sie bekämpfen kann. Rowohlt Taschenbuch Verlag 2013

2 Weidner K et al. (2007): Multiprofessionelles Netzwerk Schwangerschaft und Wochenbett. In: Beiträge der 36. Jahrestagung der Deutschen Gesellschaft für Psychosomatische Frauenheilkunde und Geburtshilfe e.V. MabuseVerlag 2008, 151-160

3 Schuster D (2014): Psychosomatisches Verständnis - ein wichtiger Bestandteil der Hebammenarbeit . Die Hebamme 2014; 27 (2): 92-96

4 Bergner A (2005): Unerwarteter Verlust und neue Hoffnung: Fehlgeburten, ihre Verarbeitung und die Folgen für eine neue Schwangerschaft. Dissertationsschrift, Institut für Psychologie der Humboldt-Universität zu Berlin.

5 Fertl K et al. (2007): Ausprägungen und Auswirkungen von Ängsten in einer Schwangerschaft nach Fehlgeburtserfahrung. In: Beiträge der 36. Jahrestagung der Deutschen Gesellschaft für Psychosomatische Frauenheilkunde und Geburtshilfe e.V. Mabuse-Verlag 2008, 139-150

6 Wolter H (2011) : Gute Hoffnung oder in großer Angst? - Folgeschwangerschaften nach dem Verlust eines Kindes. In: Beiträge der 40. Jahrestagung der Deutschen Gesellschaft für Psychosomatische Frauenheilkunde und Geburtshilfe e.V. Mabuse-Verlag 2012, 95-97

7 Janssen $\mathrm{H}$, Cuisinier M, Hoogduin K, Graauw K (1996): Controlled prospective study on the mental health of women following pregnancy loss. American Journal of Psychiatry 153: 226-230

8 Rauchfuß M, Trautmann K (1997): Ängste nach Fehl-, Früh-oder Totgeburten und ihr Einfluss auf den Schwangerschaftsverlauf. Geburtshilfe und Frauenheilkunde 1997; 142: 1176-1183

9 Klein A, Rohde A (2010): Wohin mit meiner Angst? Psychologische Basisinterventionen bei Frauen mit Ängsten nach habituellen Aborten. In: Beiträge der 39. Jahrestagung der Deutschen 
Gesellschaft für Psychosomatische Frauenheilkunde und Geburtshilfe e.V. Mabuse-Verlag 2011, 116-124

10 Hellmann V (2009): Beratung in der Schwangerschaft. Vortrag beim Curriculum für Psychosomatische Grundversorgung Dresden, 2009

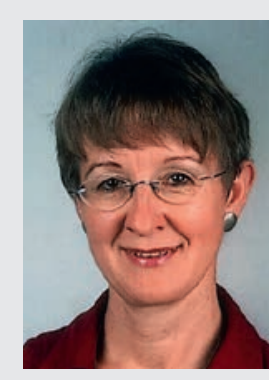

Dr. med. Dorothea Schuster Frauenärztliche Gemeinschaftspraxis Rudolf-Renner-Str. 37

01159 Dresden

E-Mail: doschuster@t-online.de 